\title{
O efeito das equipes multiprofissionais em saúde no brasil em atividades de cuidado com o diabetes
}

The effect of multiprofessional health teams in Brazil on diabetes care activities

El efecto de los equipos multiprofesionales en salud en Brasil en actividades de cuidado con la diabetes

Daniel Leonardo Ferreira1*, Elisabete Aparecida Mantovani Rodrigues de Resende ${ }^{1}$, Ana Luiza Rosa Lucas ${ }^{1}$, Adryelle Cristina Ferreira Silva ${ }^{1}$, Sarah Santiago Lenci ${ }^{1}$, Sarah Gazarra Ferreira Silva ${ }^{1}$, Fausto Jorge de Almeida Júnior ${ }^{1}$, Flávia Hermano Rodrigues dos Santos ${ }^{1}$, Flávia Mansur Passarelli ${ }^{1}$, Gabriela Marques Bortolotto ${ }^{1}$, Isabela de Paula Rezende ${ }^{1}$, Jussara da Cruz Jardim¹, Roberta Garcia Leal Veiga Gonçalves ${ }^{1}$, Lauana Andrade Messias²

\section{RESUMO}

Objetivos: $O$ artigo trata-se de uma revisão bibliográfica e tem como objetivo conhecer as estratégias educativas da equipe multiprofissional com pacientes diabéticos, de maneira a contribuir com a promoção em saúde. Metodologia: Revisão sistemática da literatura, baseados nas estratégias de promoção à saúde através da equipe multiprofissional em Diabetes. Foram escolhidos artigos publicados entre 1999 e 2017. As bases de dados foram LILACS, SciELO, e PUBMED. A pesquisa foi efetuada com os descritores: Equipe multiprofissional, promoção, saúde, Diabetes. Resultados e discussão: Foram coletados 35 artigos. Todos os trabalhos continham pelo menos uma prática de prevenção e cuidado com o Diabetes, listados em tabela. Conclusão: $O$ elo entre o conhecimento teórico-prático dos profissionais de saúde, a imposição de estratégias de cuidado com o Diabetes, e a participação efetiva dos usuários e familiares, potencializam os efeitos benéficos no tratamento dessa doença.

Palavras-chaves: Diabetes, Equipe Multiprofissional, Promoção, Saúde.

\begin{abstract}
Objective: The article is a bibliographical review and aims to know the educational strategies of the multiprofessional team with diabetic patients, in order to contribute to health promotion. Methods: Systematic review of the literature, based on strategies for health promotion through the multiprofessional team in Diabetes. Articles were published between 1999 and 2017. The databases were LILACS, SciELO, and PUBMED. The research was carried out with the following descriptors: Multiprofessional team, promotion, health, Diabetes. Finds and discussion. A total of 35 articles were collected. All jobs contained at least one Diabetes prevention and care practice listed in the table. Conclusion: The link between theoretical and practical knowledge of health professionals, the imposition of Diabetes care strategies, and the effective participation of users and their families, potentiate the beneficial effects in the treatment of this disease.
\end{abstract}

Keywords: Diabetes, Multiprofessional Team, Promoction, Health.

1 Universidade Federal do Triângulo Mineiro (UFTM), Uberaba - MG. * E-mail: biologia.daniel89@gmail.com
${ }_{2}$ Faculdade de Ciências Médicas da Universidade Estadual de Campinas (UNICAMP), Campinas-SP.

SUBMETIDO EM: 10/2018

ACEITO EM: 11/2018

PUBLICADO EM: 12/2018 


\section{RESUMEM}

Objetivos: El artículo trata de una revisión bibliográfica y tiene como objetivo conocer las estrategias educativas del equipo multiprofesional con pacientes diabéticos, con objeto de contribuir con la promoción de la salud. Metodologia: Revisión sistemática de la literatura, basadas en las estrategias de promoción de la salud a través del equipo multiprofesional en la Diabetes. Se eligieron artículos publicados entre 1999 y 2017. Las bases de datos fueron LILACS, SciELO, y PUBMED. La investigación fue efectuada con los descriptores: equipo multiprofesional, promoción, salud, diabetes. Resultados y discusión: Se recopilaron 35 artículos. Todos los trabajos contenían por lo menos una práctica de prevención y cuidado con la Diabetes, listados en tabla. Conclusion: El enlace entre el conocimiento teórico-práctico de los profesionales de la salud, la imposición de estrategias de cuidado con la Diabetes, y la participación efectiva de los usuarios y familiares, potencian los efectos beneficiosos en el tratamiento de esta enfermedad.

Palabras-claves: Diabetes, Equipo Multiprofesional, Promoción, Salud.

\section{INTRODUÇÃO}

O Diabetes Mellitus (DM) consiste em uma condição patológica causada pela ausência ou insuficiência de insulina no organismo. É caracterizado pela hiperglicemia, a qual leva a diversas complicações para o paciente (SBD, 2017). As principais complicações crônicas são as vasculopatias (micro e macroangiopatias) e neuropatias (autonômicas e sensitivo-motoras). Representando as microangiopatias estão a nefropatia, retinopatia e neuropatia. Entre as macroangiopatias destacam-se a Doença Arterial Coronariana (DAC), Acidente Vascular Encefálico (AVE) e a Doença Vascular Periférica (DVP), principalmente o pé diabético. O DM tem relação com a precocidade da DCV, adiantando sua ocorrência em torno de 15 anos, nos indivíduos diabéticos não controlados (CARVALHO, 2016). Sua incidência vem aumentando de modo preocupante nos países em desenvolvimento, tanto em adultos quanto em adolescentes, e estima-se um aumento de $60 \%$ da prevalência na população adulta acima de 30 anos em 2025, sendo mais significativa na faixa dos 45 aos 64 anos (GROSS, 2002). Estima que a diabetes seja a 7. - principal causa de morte em 2030 (OMS, 2014).

O DM é classificado como tipo 1, (ausência ou déficit na produção de insulina), tipo 2 (resistência insulínica), outros tipos e gestacional (condição associada à resistência insulínica e gravidez).

O trabalho de prevenção contra o DM ainda é o mais eficaz. Pode ocorrer de duas formas, contra o início do DM (prevenção primária) ou de suas complicações agudas ou crônicas (prevenção secundária) (SBD, 2017). Tais estratégias consistem em Educação em Saúde, grupos de orientação preventiva, reuniões com profissionais da saúde, entre outros.

Sendo assim, é importante que ocorra uma intervenção multiprofissional em saúde, para que os dados epidêmicos do DM sejam reduzidos. Pacientes diabéticos, muitas vezes, desconhecem a gravidade dessa doença, por falta de informação necessária. Frequentemente, os atendimentos clínicos à diabéticos acontecem com um tempo insuficiente para sanar todas as dúvidas do enfermo, até pela alta demanda de agendamentos feitos pelo Sistema Único de Saúde (CARVALHO, 2016)

O trabalho de orientação e prevenção realizado por equipes multiprofissionais em pacientes diabéticos está bem estabelecido. Os principais fatores avaliados nesta prevenção são listados como mudança de estilo de vida, redução de estresse físico e emocional, correção de peso corporal (perda ou ganho se necessário) e exercício físico. O trabalho de prevenção é feito principalmente em unidades básicas de saúde (UBS), por nutricionistas, enfermeiros, fisioterapeutas e educadores em Saúde, dentro de um programa atenção primária à saúde. Muitos estudos demonstraram que mudanças no estilo de vida são efetivas em retardar ou prevenir o diabetes, oferecendo uma qualidade de vida maior aos pacientes. (D'AGOSTINO, 2008).

O artigo trata-se de uma revisão bibliográfica e tem como objetivo conhecer as estratégias educativas da equipe multiprofissional com pacientes diabéticos, de maneira a contribuir com a promoção em saúde. 


\section{MÉTODOS}

Trata-se de uma revisão sistemática da literatura, conduzida por meio da síntese de resultados de estudos originais, quantitativos ou qualitativos, baseados nas estratégias de promoção à saúde através da equipe multiprofissional em Diabetes.

Para tanto, foram escolhidos artigos publicados entre 1999 e 2017. As bases de dados foram LILACS, SciELO, e PUBMED. A pesquisa foi efetuada com os descritores: Equipe multiprofissional, promoção, saúde, Diabetes. Foram excluídos artigos anteriores à 2006 e que não contavam com intervenção direta com pacientes portadores de DM ou doenças crônicas comuns.

\section{RESULTADOS E DISCUSSÃO}

Foram coletados 35 artigos, estes obtidos nas bases de dados conforme mostra a quadro 1. Todos os trabalhos continham pelo menos uma prática de prevenção e cuidado com o Diabetes, onde foram listados os principais na tabela 1. Observou-se nos trabalhos, principalmente, os impactos sobre os diferentes fatores que interferem no processo de cuidado com o Diabetes. As ações multiprofissionais permitiram a possibilidade da prática de um profissional se reconstruir na prática do outro, ambos sendo transformados para a intervenção na realidade em que estão inseridos.

Quadro 1 - Demonstração panorâmica do estudo.

\begin{tabular}{|c|c|c|c|}
\hline Autores & Profissionais envolvidos & Intervenção & Considerações importantes \\
\hline $\begin{array}{l}\text { Campos, } \\
1999\end{array}$ & $\begin{array}{l}\text { As equipes obedeceriam a } \\
\text { uma composição } \\
\text { multiprofissional }\end{array}$ & $\begin{array}{l}\text { Novo sistema de referência } \\
\text { entre profissionais e usuários, } \\
\text { equipes de referência e } \\
\text { trabalho de apoio matricial }\end{array}$ & $\begin{array}{l}\text { Propõe a criação de um modelo de } \\
\text { atendimento mais singularizado e mais } \\
\text { personalizado, através da construção de } \\
\text { planos terapêuticos singulares }\end{array}$ \\
\hline $\begin{array}{l}\text { Ferraz et.al., } \\
\quad 2000\end{array}$ & $\begin{array}{l}\text { Equipe multiprofissional } \\
\text { em Saúde composta por } \\
\text { Médicos e Enfermagem }\end{array}$ & $\begin{array}{l}\text { Conscientização e Educação } \\
\text { em Saúde }\end{array}$ & $\begin{array}{l}\text { É essencial assegurar que os pacientes } \\
\text { com diabetes estejam em condições de } \\
\text { adquirir conhecimentos e aptidões para o } \\
\text { autocuidado. }\end{array}$ \\
\hline $\begin{array}{l}\text { Leite et al., } \\
\quad 2001\end{array}$ & $\begin{array}{l}\text { Médicos, enfermeiros, } \\
\text { assistente social e } \\
\text { nutricionistas }\end{array}$ & $\begin{array}{l}\text { Entrevista com a assistente } \\
\text { social, seguida de avaliação } \\
\text { pela enfermagem, } \\
\text { nutricionista e } \\
\text { endocrinologista }\end{array}$ & $\begin{array}{l}\text { A abordagem multiprofissional é } \\
\text { considerada ideal para o atendimento ao } \\
\text { diabético devido à complexidade da } \\
\text { consulta, que deve abordar muitos } \\
\text { detalhes do tratamento medicamentoso, } \\
\text { dietoterápico e educação destes } \\
\text { pacientes na prevenção das } \\
\text { complicações crônicas. }\end{array}$ \\
\hline $\begin{array}{l}\text { Torres et.al., } \\
2003\end{array}$ & $\begin{array}{l}\text { Profissionais da saúde } \\
\text { como, enfermeiro, } \\
\text { endocrinologista, } \\
\text { nutricionista terapeuta } \\
\text { ocupacional, educador } \\
\text { físico e o paciente de } \\
\text { diabetes mellitus }\end{array}$ & $\begin{array}{l}\text { Jogos educativos em } \\
\text { ambulatório de } \\
\text { especialidades, no caso } \\
\text { Diabetes Mellitus }\end{array}$ & $\begin{array}{l}\text { A estratégia de uso jogo educativos } \\
\text { proporciona benefícios para o paciente } \\
\text { se autoconhecer e o profissional } \\
\text { conhece-lo também }\end{array}$ \\
\hline Ceccin, 2004 & Profissionais da saúde & $\begin{array}{l}\text { Proposta de uma ética entre } \\
\text { disciplinar à estética } \\
\text { multiprofissional do trabalho e } \\
\text { educação da equipe de } \\
\text { saúde. }\end{array}$ & $\begin{array}{l}\text { A ética resulta em uma mestiçagem e } \\
\text { aprendizado dos problemas de saúde, } \\
\text { como experimentados em situações de } \\
\text { vida e terapêuticas. }\end{array}$ \\
\hline $\begin{array}{l}\text { Albuquerque } \\
\text { PC e Stotz } \\
\text { EN, } 2004\end{array}$ & $\begin{array}{l}\text { Profissionais de saúde } \\
\text { (PSF) }\end{array}$ & $\begin{array}{l}\text { Ações educativas em que, } \\
\text { tanto profissionais como } \\
\text { usuários aprendem e } \\
\text { ensinam, numa construção } \\
\text { dialógica do conhecimento }\end{array}$ & $\begin{array}{l}\text { A educação popular contribui para a } \\
\text { inclusão de novos atores e abertura de } \\
\text { canais de participação no nível local. }\end{array}$ \\
\hline $\begin{array}{l}\text { Santos et } \\
\text { al.,2005 }\end{array}$ & $\begin{array}{l}\text { Equipe Médica e de } \\
\text { Enfermagem }\end{array}$ & $\begin{array}{l}\text { Analise do discurso de áudio } \\
\text { de pacientes diabéticos }\end{array}$ & $\begin{array}{l}\text { Recomenda-se qualificar o paciente } \\
\text { diabético como seu principal cuidador. }\end{array}$ \\
\hline
\end{tabular}


Continuação do Quadro 1.

\begin{tabular}{|c|c|c|c|}
\hline Autores & Profissionais envolvidos & Intervenção & Considerações importantes \\
\hline $\begin{array}{l}\text { Barros et } \\
\text { al.,2006 }\end{array}$ & $\begin{array}{l}\text { Docentes da área da } \\
\text { saúde. }\end{array}$ & $\begin{array}{l}\text { Projeto político-pedagógico, } \\
\text { visando a formação do } \\
\text { profissional baseado na } \\
\text { necessidade de se manter } \\
\text { interface com outras áreas do } \\
\text { conhecimento e com as } \\
\text { políticas públicas. }\end{array}$ & $\begin{array}{l}\text { O ensino de Educação em Saúde, } \\
\text { Interdisciplinaridade e Políticas Públicas } \\
\text { oferece uma contribuição valiosa para a } \\
\text { reflexão de educadores e educandos } \\
\text { comprometidos com o fortalecimento das } \\
\text { políticas de saúde. }\end{array}$ \\
\hline $\begin{array}{l}\text { Paixão e } \\
\text { Castro, } 2006\end{array}$ & $\begin{array}{l}\text { Alunos estagiários de } \\
\text { Enfermagem, Nutrição e } \\
\text { diversas áreas }\end{array}$ & Grupos de Sala de Espera & $\begin{array}{l}\text { Índice significativo de benefício para o } \\
\text { usuário }\end{array}$ \\
\hline $\begin{array}{c}\text { Araújo } \\
\text { Rocha, } 2007\end{array}$ & $\begin{array}{l}\text { Equipe de Saúde da } \\
\text { família }\end{array}$ & $\begin{array}{l}\text { Trabalho das equipe em } \\
\text { saúde que atuam no } \\
\text { Programa de Saúde da } \\
\text { Família (PSF), em especial } \\
\text { em Natal-RN }\end{array}$ & $\begin{array}{l}\text { Nova dimensão do trabalho em equipe no } \\
\text { PSF gera uma divisão de } \\
\text { responsabilidades do cuidado entre os } \\
\text { membros da equipe. }\end{array}$ \\
\hline $\begin{array}{l}\text { Cardoso et } \\
\text { al.,2007 }\end{array}$ & $\begin{array}{l}\text { Equipe de assistência ao } \\
\text { paciente- interdisciplinar }\end{array}$ & $\begin{array}{l}\text { Discussão sobre a proposta } \\
\text { de interdisciplinaridade no } \\
\text { âmbito da saúde }\end{array}$ & $\begin{array}{l}\text { A compreensão do movimento } \\
\text { interdisciplinar em que a uma troca de } \\
\text { saberes entre as diferentes disciplinas }\end{array}$ \\
\hline $\begin{array}{l}\text { Otero et al } \\
., 2008\end{array}$ & $\begin{array}{l}\text { Enfermeiros, Médicos } \\
\text { Endocrinologistas, } \\
\text { Nutricionistas, Psicólogos, } \\
\text { Educador Físico e alunos } \\
\text { de } \\
\text { Graduação em } \\
\text { Enfermagem e Psicologia }\end{array}$ & $\begin{array}{l}\text { Estudo experimental, } \\
\text { comparativo, do tipo antes e } \\
\text { depois }\end{array}$ & $\begin{array}{l}\text { É necessário ensinar ao paciente sobre } \\
\text { sua doença e, além disso, acompanhar } \\
\text { seu tratamento. }\end{array}$ \\
\hline $\begin{array}{l}\text { Ribas } \\
\text { et.al.,2008 }\end{array}$ & Equipe de enfermagem & $\begin{array}{l}\text { Estudo utilizando a técnica de } \\
\text { incidentes críticos }\end{array}$ & $\begin{array}{l}\text { A importância de um investimento } \\
\text { adequado para desenvolvimento de } \\
\text { atividades de promoção à saúde. }\end{array}$ \\
\hline $\begin{array}{l}\text { Ferreira et } \\
\text { al.,2011 }\end{array}$ & $\begin{array}{l}\text { Enfermeiros, Psicólogos, } \\
\text { Educadores Físicos e } \\
\text { Nutricionistas }\end{array}$ & $\begin{array}{l}\text { Grupos de orientação } \\
\text { preventiva multiprofissional }\end{array}$ & $\begin{array}{l}\text { Diferentes modos de lidar com os } \\
\text { desafios da doença no cotidiano, } \\
\text { influenciam diretamente a adesão ao } \\
\text { tratamento e intervenção }\end{array}$ \\
\hline $\begin{array}{l}\text { Frigo et al., } \\
2012\end{array}$ & $\begin{array}{l}\text { Medicina, Enfermagem e } \\
\text { Equipe de Saúde da } \\
\text { Família }\end{array}$ & $\begin{array}{l}\text { Atendimento multiprofissional } \\
\text { em atenção básica }\end{array}$ & $\begin{array}{l}\text { A prática educativa apresenta-se como a } \\
\text { melhor maneira de conscientizar a } \\
\text { pessoa com diabetes. }\end{array}$ \\
\hline $\begin{array}{l}\text { Assis et al, } \\
2012\end{array}$ & $\begin{array}{l}\text { Medicina e equipe } \\
\text { multiprofissional (não } \\
\text { especificada) }\end{array}$ & $\begin{array}{l}\text { Revisão da literatura. } \\
\text { Observa ações da equipe de } \\
\text { saúde em UBS e USF. }\end{array}$ & $\begin{array}{l}\text { A proximidade do paciente com doenças } \\
\text { crônicas com os serviços de saúde } \\
\text { diminui os riscos de morte. }\end{array}$ \\
\hline $\begin{array}{l}\text { Boeira et } \\
\text { al,2012 }\end{array}$ & $\begin{array}{l}\text { Técnicos e auxiliares de } \\
\text { enfermagem, enfermeiros, } \\
\text { médicos, psicólogos, } \\
\text { dentistas, assistente } \\
\text { social, docentes do Curso } \\
\text { de Fisioterapia, Nutrição e } \\
\text { Enfermagem e } \\
\text { acadêmicos de } \\
\text { Enfermagem }\end{array}$ & Oficinas educativas & $\begin{array}{l}\text { Há o aperfeiçoamento do processo de } \\
\text { educação em saúde, quando está } \\
\text { associado aos cuidados interdisciplinares } \\
\text { para diabetes. A prática educativa } \\
\text { apresenta-se uma maneira efetiva de } \\
\text { conscientizar a pessoa com DM sobre a } \\
\text { importância da melhora dos hábitos } \\
\text { diários e do seu próprio cuidado. }\end{array}$ \\
\hline $\begin{array}{l}\text { Carvalho, } \\
2012\end{array}$ & $\begin{array}{l}\text { Enfermagem e Gestores } \\
\text { do grupo Hiperdia }\end{array}$ & $\begin{array}{l}\text { Revisão da literatura. } \\
\text { Observa ações da equipe de } \\
\text { Enfermagem no programa } \\
\text { Hiperdia }\end{array}$ & $\begin{array}{l}\text { A educação em saúde é um dos mais } \\
\text { importantes fatores para minimizar as } \\
\text { complicações destas doenças. }\end{array}$ \\
\hline Neves, 2012 & Médicos e Enfermeiros & $\begin{array}{l}\text { Revisão da literatura sobre a } \\
\text { atuação interdisciplinar entre } \\
\text { enfermeiros e outros } \\
\text { profissionais da saúde. }\end{array}$ & $\begin{array}{l}\text { Apesar do foco ser a equipe de } \\
\text { Enfermagem, é necessárias ações } \\
\text { coletivas para melhores resultados }\end{array}$ \\
\hline $\begin{array}{c}\text { Alves e } \\
\text { Pinto, } 2013\end{array}$ & $\begin{array}{l}\text { Assistente social, } \\
\text { enfermeiro, educador } \\
\text { físico, fisioterapeuta, } \\
\text { nutricionista, psicólogo e } \\
\text { terapeuta ocupacional }\end{array}$ & $\begin{array}{l}\text { Revisão integrativa da } \\
\text { literatura sobre estratégias de } \\
\text { intervenção utilizadas por } \\
\text { equipes multiprofissionais na } \\
\text { atenção à saúde de } \\
\text { adolescentes }\end{array}$ & $\begin{array}{l}\text { A produção científica recente em ações } \\
\text { multiprofissionais relacionadas á saúde } \\
\text { do adolescente está voltada aos relatos } \\
\text { de experiências }\end{array}$ \\
\hline
\end{tabular}


Continuação do Quadro 1.

\begin{tabular}{|c|c|c|c|}
\hline Autores & Profissionais envolvidos & Intervenção & Considerações importantes \\
\hline $\begin{array}{l}\text { Navarro et } \\
\text { al., } 2013\end{array}$ & $\begin{array}{l}\text { Todos profissionais das } \\
\text { ESF's }\end{array}$ & $\begin{array}{l}\text { Reflexões sobre as práticas } \\
\text { interdisciplinares em } \\
\text { pacientes com doenças } \\
\text { crônicas }\end{array}$ & $\begin{array}{l}\text { Enfatiza a alta significância do trabalho } \\
\text { em equipe realizado em sintonia com a } \\
\text { família do atendido. }\end{array}$ \\
\hline $\begin{array}{l}\text { Neto et al., } \\
2013\end{array}$ & $\begin{array}{l}\text { Todos os profissionais da } \\
\text { atenção primária à saúde }\end{array}$ & $\begin{array}{l}\text { Revisão da literatura. } \\
\text { Observa ações da equipe de } \\
\text { saúde na Esfera de Atenção } \\
\text { Primária }\end{array}$ & $\begin{array}{l}\text { A Educação em Saúde é a responsável } \\
\text { para que o paciente monitore seus riscos } \\
\text { e controle sua doença. }\end{array}$ \\
\hline $\begin{array}{l}\text { Juniro et al., } \\
2013\end{array}$ & $\begin{array}{l}\text { Medicina, Enfermagem e } \\
\text { Psicologia }\end{array}$ & $\begin{array}{l}\text { Educação em saúde; } \\
\text { reuniões e palestras }\end{array}$ & $\begin{array}{l}\text { Importância adequada do diálogo entre } \\
\text { todos os componentes de uma equipe } \\
\text { multidisciplinar. }\end{array}$ \\
\hline $\begin{array}{l}\text { Castro et al., } \\
2014\end{array}$ & $\begin{array}{l}\text { Docentes, Residência } \\
\text { Multiprofissional, técnicos, } \\
\text { auxiliares administrativos } \\
\text { e alunos em nível de } \\
\text { graduação e pós- } \\
\text { graduação }\end{array}$ & $\begin{array}{l}\text { Educação em saúde; } \\
\text { reuniões de pacientes } \\
\text { diabéticos }\end{array}$ & $\begin{array}{l}\text { Ocorreu significativa melhora na } \\
\text { qualidade de vida dos indivíduos } \\
\text { atendidos. }\end{array}$ \\
\hline $\begin{array}{l}\text { Cruz et } \\
\text { al.,2014 }\end{array}$ & Enfermagem & $\begin{array}{l}\text { Orientação preventiva com } \\
\text { mães }\end{array}$ & $\begin{array}{l}\text { A importância da singularidade da } \\
\text { relação estabelecida entre a mãe e seu } \\
\text { filho }\end{array}$ \\
\hline $\begin{array}{c}\text { Sousa et.al } \\
., 2014\end{array}$ & $\begin{array}{l}\text { Enfermagem, Medicina e } \\
\text { Nutrição }\end{array}$ & $\begin{array}{l}\text { Controle glicêmico em } \\
\text { pacientes com Diabetes } \\
\text { Gestacional }\end{array}$ & $\begin{array}{l}\text { Pacientes gestantes com DMG podem } \\
\text { realizar um bom controle glicêmico } \\
\text { através de suporte da equipe } \\
\text { multiprofissional. }\end{array}$ \\
\hline $\begin{array}{c}\text { Corrêa et al } \\
., 2014\end{array}$ & $\begin{array}{l}\text { Todas as áreas da } \\
\text { Residência } \\
\text { Multiprofissional em } \\
\text { Saúde. Ênfase nos } \\
\text { Educadores Físicos }\end{array}$ & Atendimento multiprofissional & $\begin{array}{l}\text { O trabalho da equipe multiprofissional } \\
\text { contribui no controle de doenças } \\
\text { crônicas. }\end{array}$ \\
\hline $\begin{array}{l}\text { Camara e } \\
\text { Forti, } 2015\end{array}$ & Psicologia e Medicina & $\begin{array}{l}\text { Revisão sobre a importância } \\
\text { da atuação da equipe } \\
\text { multidisciplinar }\end{array}$ & $\begin{array}{l}\text { Integração entre diversas áreas da saúde } \\
\text { no controle do DM. }\end{array}$ \\
\hline $\begin{array}{l}\text { Santos } \\
\text { et.al.,2015 }\end{array}$ & Enfermagem & Palestras do Hiperdia & $\begin{array}{l}\text { A importância de se disseminar } \\
\text { informações amplas sobre o processo do } \\
\text { autocuidado. }\end{array}$ \\
\hline $\begin{array}{l}\text { Coqueiro } \\
\text { et.al.,2015 }\end{array}$ & Todas as áreas da saúde & $\begin{array}{l}\text { Revisão bibliográfica sobre } \\
\text { estratégias de Educação em } \\
\text { Saúde }\end{array}$ & $\begin{array}{l}\text { É essencial para o paciente diabético } \\
\text { uma completa intervenção das } \\
\text { Estratégias de Saúde. }\end{array}$ \\
\hline $\begin{array}{l}\text { Gritti } \\
\text { et.al.,2015 }\end{array}$ & $\begin{array}{l}\text { Equipe multiprofissional } \\
\text { de saúde composta por } \\
\text { médicos, enfermeiros, } \\
\text { educadores físicos, } \\
\text { nutricionistas entre outros }\end{array}$ & $\begin{array}{l}\text { Entrevista e comparação de } \\
\text { dados clínicos pré e pós } \\
\text { entrevistas. }\end{array}$ & $\begin{array}{l}\text { É necessário melhorar as estratégias de } \\
\text { prevenção em saúde para o paciente } \\
\text { diabético. }\end{array}$ \\
\hline $\begin{array}{l}\text { Campos et.al } \\
\quad, 2016\end{array}$ & $\begin{array}{l}\text { Medicina e equipe } \\
\text { multiprofissional (não } \\
\text { especificada) }\end{array}$ & $\begin{array}{l}\text { Cirurgia metabólica e } \\
\text { acompanhamento } \\
\text { multiprofissional }\end{array}$ & $\begin{array}{l}\text { Cirurgia metabólica, acompanhada de } \\
\text { intervenção multiprofissional, apresenta } \\
\text { menores riscos do que a bariátrica. }\end{array}$ \\
\hline $\begin{array}{l}\text { Ales-Peres et } \\
\quad \text { al., } 2016\end{array}$ & $\begin{array}{l}\text { Equipe multidisciplinar } \\
\text { (não especifica) }\end{array}$ & $\begin{array}{l}\text { Palestras, orientação } \\
\text { individual, mudança cardápio } \\
\text { da escola, dados } \\
\text { antropométricos, dados } \\
\text { HbA1c, incentivo de atividade } \\
\text { física, }\end{array}$ & $\begin{array}{l}\text { Índice alto de inatividade física, fatores } \\
\text { psicológicos relacionados ao descontrole } \\
\text { glicêmico. }\end{array}$ \\
\hline $\begin{array}{l}\text { Molmester et } \\
\text { al., } 2016\end{array}$ & $\begin{array}{l}\text { Equipe multiprofissional } \\
\text { completa }\end{array}$ & $\begin{array}{l}\text { Palestras e procedimentos } \\
\text { clínicos }\end{array}$ & $\begin{array}{l}\text { A importância de um olhar crítico da } \\
\text { Nutrição para desenvolvimento de uma } \\
\text { dieta saudável. }\end{array}$ \\
\hline $\begin{array}{l}\text { Ramalho et } \\
\text { al., } 2017\end{array}$ & Equipe multiprofissional & Palestras de intervenção & $\begin{array}{l}\text { O trabalho feito pelas Unidades de saúde } \\
\text { é essencial para o paciente com } \\
\text { Diabetes. }\end{array}$ \\
\hline
\end{tabular}

Fonte: Dados da pesquisa, 2018. 
A grande amplitude dos estudos analisados mostrou a importância das práticas educativas. $O$ que aprofundou essa discussão de forma mais clara, destacando a visão holística da educação e promoção em saúde foi o de (CARVALHO, 2013). Para ilustrar tal questão, destaca-se uma tríade importante no controle do Diabetes Mellitus. A família, rede de apoio e comunidade são indispensáveis, conforme mostra Torres. (TORRES, 2003). Ele cita a intervenção ocorrida no Ambulatório Borges da Costa (SEEM/HC/UFMG), onde foi realizado um Programa Educativo através de Jogos com pessoas diabéticas. $O$ jogo incentivava a presença de um acompanhante, seja da família ou alguém da vizinhança

Percebe-se que, com o passar dos anos, as intervenções multiprofissionais no controle das doenças crônicas foram sofrendo alterações. Inicialmente eram realizadas por médicos e enfermeiros, conforme mostra Campos. (CAMPOS,1999) e depois foi aprimorada, com um novo sistema de referência entre profissionais e usuários dos serviços de saúde, com a formação de equipes básicas direcionadas à objetivos específicos, observadas nas práticas recentes. (CECCIM 2004). Ele reforça a importância de aproximar o usuário do sistema, mas ressalta que acima do trabalho em grupo, deve existir a especificidade de cada profissional, o que se trata de uma visão moderna. Já Duarte e Gonçalves, (2011) trazem a ideia de que o saber técnico-científico desses profissionais são fundamentais para que os mesmos possam intervir no processo saúde-doença. O indivíduo diabético pode ter várias complicações, e cada profissional contribui para evitá-las.

Os artigos que trazem atividades educativas sugerem que a equipe multiprofissional ideal deve ser composta por endocrinologista, enfermeiro, auxiliar de enfermagem, cirurgião vascular, cirurgião plástico, ortopedista, dermatologista, fisioterapeuta, podólogo e técnico ortopédico, para que seja oferecida uma consulta específica de diabetes nas unidades básicas de saúde. Entretanto, sabe-se que a atual realidade está longe da ideal.(REZENDE, 2009). Ele trouxe a ideia de que a equipe multiprofissional é efetiva no controle de riscos, incentivando um estilo de vida saudável e estabelecendo métodos de cuidado do DM. Corroborando com Torres, o estudo de Cardoso (2009), confirma a importância da educação em saúde através de práticas educativas. Porém, acrescenta que é necessário estimular não só a interdisciplinaridade, mas também as experiências vivenciadas em diversas etapas da vida, com o objetivo de auxiliar o tratamento do paciente diabético. (CARDOSO, 2009)

É importante contextualizar as práticas ocorridas no Brasil, com a de diversos países. O cuidado com o Diabetes através de equipe multiprofissional merece destaque. Na Europa, em países como Reino Unido, Holanda e Dinamarca as práticas coletivas visam a amenização dos riscos, triagem e cuidado preventivo para evitar as complicações tardias da doença. O Canadá merece destaque, devido as suas políticas de saúde pública e intervenção sobre as doenças crônicas. O país apresenta 15 sistemas de saúde distintos, mas que atuam focados na prevenção e práticas multiprofissionais modernas e eficientes. Um dos exemplos de modelos de intervenção é o desenvolvimento do "Modelo Michigan para Diabetes Tipo 2" qualidade de vida e custos. Ele é projetado para avaliar a eficácia e custo-utilidade de estratégias alternativas para a prevenção e tratamento de diabetes tipo 2. (HERMAN, 2015). Essa avaliação permite nortear as práticas clínicas e multiprofissionais e seu custo-benefício ao Estado. No Brasil, embora exista limitações visíveis e problemas de saúde pública como a falta de profissionais, muito tem-se feito para melhorar a atividade das equipes multidisciplinares..

\section{CONSIDERAÇÕES FINAIS}

A intervenção da equipe multiprofissional no tratamento do DM traz ações transformadoras que favorecem o paciente. O elo entre o conhecimento teórico-prático dos profissionais de saúde, a imposição de estratégias de cuidado com o Diabetes, e a participação dos efetiva dos usuários e familiares, potencializam os efeitos benéficos no tratamento dessa doença. 


\section{REFERÊNCIAS}

1. ALBUQUERQUE PC, STOTZ EN. Popular education in primary care: in search of comprehensive health care, Interface - Comunic., Saúde, Educ., v.8, n.15, p.259-74, mar/ago 2004.

2. ALVES PAS, PINTO AP. Intervenções e práticas multiprofissionais. Rev. Ter. Ocup. Univ. São Paulo, 2013 maio/ago, 24(2);168-73

3. ANTONELA FA, SIQUEIRA BA, SANDRA RG et al. Doença Cardiovascular no Diabetes Mellitus: Análise dos Fatores de Risco Clássicos e Não-Clássicos. Arquivo Brasileiro de Endocrinologia e Metabolismo, v.51(2), 2007.

4. ARAUJO MBS, ROCHA PM. Trabalho em equipe: um desafio para a consolidação da estratégia de saúde da família. Rev Ciência e Saúde Coletiva 455-464. 2007.

5. BRASIL. Ministério da Saúde. Prevenção clínica de doenças cardiovasculares, cerebrovasculares e renais. Brasília: MS, 2006. CAMPOS, F. C. C. de; FARIA, H. P. de; SANTOS, M. A. dos.

6. CARDOSO JP, VILELA ABA, SOUZA NR et al. Formação interdisciplinar: efetivando propostas de promoção da saúde no Sus. RBPS 2007; 20 (4) : 252-258

7. CARVALHO FPB, SIMPSON CA, QUEIROZ TA. Revista de enfermagem UFPE online., Recife, v.10(2):750-5, fev, 2016

8. CORRÊA FH, NOGUEIRA VG, BEVILÁCQUA MF et al. Avaliação da secreção e resistência insulínica em indivíduos com diferentes graus de tolerância à glicose - do metabolismo normal ao diabetes mellitus. ArqBrasEndocrinolMetabol. 2007;51(9):1498-505

9. COSTA JR, R LL. Desfechos clínicos tardios de pacientes diabéticos tratados com stents farmacológicos eluidores de sirolimus ou everolimus: uma análise do registro DESIRE. Revista Brasileira de Cardiologia Invasiva, v.23 (1):17-21, 2015.

10. CRUZ DSM, COLLET N, ANDRADE EM et al . Vivências de mães de crianças diabéticas. Escola Anna Nery 21(1) 2017

11. D'AGOSTINO RB, VASAN RS, PENCINA MJLL. General cardiovascular risk profile for use in primarycare: the Framingham Heart Study. Circulation, 117 (6):743-53, 2008

12. DORES J. Insulinoterapia na diabetes mellitus tipo 2. Rev Port Cardiol. 2013;32(Supl. I):25-31

13. Elaboração do plano de ação. In: CAMPOS, F. C. C. de; FARIA, H. P. de; SANTOS, M. A. dos. Planejamento e avaliação das ações em saúde. $2^{\underline{a}}$ ed. Belo Horizonte: Nescon/UFMG, 2010. 118p

14. FRIGO LF, SILVA RM, MATTOS KM et. al. Ação educativa interdisciplinar para pacientes com diabetes na atenção básica: uma revisão bibliográfica. Rev Epidemiol Control Infect. 2012;2(4):141-143

15. GROSS JL, SILVEIRO SP, CAMARGO JL et al. Diabetes melito: diagnóstico, classificação e avaliação do controle glicêmico. Arquivo Brasileiro deEndocrinologia e Metabologia, v.46: 16-26, 2002.

16. JUUTILAINEN A, LEHTO S, ONNEMAA T et al. Type 2 Diabetes as a "Coronary Heart Disease Equivalent". Diabetes Care;v. 28(12): 2901-7,2005.

17. MARASCHIN JF, MURUSSI N, WITTER M et.al Classificação do diabete melito. Arquivo Brasileiro de Cardiologia,v.95(2) :4047,2010 .

18. MENDES EV. O cuidado das condições crônicas na atenção primária à saúde: o imperativo da consolidação da estratégia da saúde da família. Brasília: Organização Pan Americana da Saúde,2012

19. MICHELS MJ. Questionário de Atividades de Autocuidado com o Diabetes: tradução, adaptação e avaliação das propriedades psicométricas. ArqBrasEndocrinolMetab [online]. 2010, vol.54, n.7 [cited 2016-06-01], pp.644-651.

20. PREIS SR, HWANG SJ, COADY S et al. Trends in all-cause and cardiovascular disease mortality among women and men with and without diabetes mellitus in the Framingham Heart Study, 1959 to 2005. Circulation; 119(13):1728-35 2009.

21. RADIGONDA R, SOUZA LCJ, SILVA AMR. Avaliação do acompanhamento de pacientes adultos com hipertensão arterial e ou diabetes melito pela Estratégia Saúde da Família e identificação de fatores associados, Epidemiologia Serviço e Saúde, Brasília, 25(1):115-126, jan-mar, 2016.

22. REZENDE, Jofre M. Linguagem Médica, 3a. ed., Goiânia, AB Editora e Distribuidora de Livros Ltda, 2004.

23. SCHMIDTI MI, DUNCANI BB, HOFFMANNII JF et al. Prevalência de diabetes e hipertensão no Brasil baseada em inquérito de morbidade auto referida Brasil 2006. Revista de Saúde Pública,v.43(2):74-82,2009.

24. SOUSA AMS, FIUZA D, MIKAMI FC et.al. Evaluation of information retention and adherence to treatment in patients with gestational diabetes mellitus after multidisciplinary group. Rev assoc med bras 2016; 62(3):212-217

25. TORRES HC, FRANCO JL, STRADIOTO MA et. al. Avaliação estratégica de educação em grupo e individual no programa educativo em diabetes. Rev Saúde Pública 2009;43(2):291-8

26.TORRES HC, HORTALE VA, SCHALL V. A experiência de jogos em grupos operativos na educação em saúde para diabéticos. Cad. Saúde Pública, Rio de Janeiro, 19(4):1039-1047, jul-ago, 2003. 